Agency for Healthcare Research and Quality as a part of Healthcare Cost and Utilisation Project. Univariate and multivariate binomial logistic regressions were used to derive odds ratio for predictors of hip surgery. Statistical analysis was done using STATA version 13.0 (College Station, TX).

Results: NIS database from 2009-2011 contained 3538 (weighted counts in the whole US population $n=17,480$ ) patients with AS. Out of those, 190 (weighted $\mathrm{n}=934)$ had hip surgery $(5.36 \%)$. Multivariate binomial regression analysis after controlling for confounders (table 1) showed male sex (OR 2.52, Cl 1.65-3.83, $\mathrm{p}<0.001$ ) and peripheral enthesopathy (OR 8.64, $\mathrm{Cl} 2.48-30.12, \mathrm{p}<0.001$ ) to be significantly associated with hip surgery in AS patients, and an inverse relationship with inflammatory bowel disease (IBD) (OR $0.35, \mathrm{Cl} 0.16-0.76, \mathrm{p}=0.01$ ) was seen.

\begin{tabular}{lccccc}
\hline AS with Hip Surgery & $\begin{array}{c}\text { Odds } \\
\text { Ratio }\end{array}$ & $\begin{array}{c}\text { Standard } \\
\text { error }\end{array}$ & $\begin{array}{c}p \text { - } \\
\text { value }\end{array}$ & \multicolumn{2}{c}{$95 \mathrm{Cl}$} \\
& & & & $\begin{array}{c}\text { Lower } \\
\text { limit }\end{array}$ & $\begin{array}{c}\text { Upper } \\
\text { limit }\end{array}$ \\
\hline Age & 1 & 0 & 0.46 & 0.99 & 1.01 \\
Male sex & $\mathbf{2 . 5 2}$ & $\mathbf{0 . 5 4}$ & $<0.001$ & $\mathbf{1 . 6 5}$ & $\mathbf{3 . 8 3}$ \\
Non-white & 1.11 & 0.25 & 0.65 & 0.71 & 1.74 \\
Income quartile & 1.33 & 0.21 & 0.07 & 0.97 & 1.83 \\
CAD & 0.6 & 0.16 & 0.06 & 0.35 & 1.02 \\
CKD & 0.65 & 0.31 & 0.36 & 0.25 & 1.66 \\
DM & 0.64 & 0.17 & 0.09 & 0.38 & 1.07 \\
CHF & 0.14 & 0.14 & 0.05 & 0.02 & 1.04 \\
Current smoking & 0.75 & 0.18 & 0.23 & 0.46 & 1.20 \\
Obesity & 1.24 & 0.35 & 0.46 & 0.71 & 2.16 \\
Uveitis & 1.04 & 0.77 & 0.96 & 0.25 & 4.43 \\
IBD & $\mathbf{0 . 3 5}$ & $\mathbf{0 . 1 4}$ & $\mathbf{0 . 0 1}$ & $\mathbf{0 . 1 6}$ & $\mathbf{0 . 7 6}$ \\
Psoriasis & 0.85 & 0.64 & 0.84 & 0.2 & 3.74 \\
Peripheral & $\mathbf{8 . 6 4}$ & $\mathbf{5 . 5}$ & $<0.001$ & $\mathbf{2 . 4 8}$ & $\mathbf{3 0 . 1 2}$ \\
enthesopathy & & & & & \\
Constant & 0.04 & 0.01 & $<0.001$ & 0.02 & 0.06 \\
\hline
\end{tabular}

Conclusions: Our study found male sex and patients with peripheral enthesopathy to have higher odds of severe hip disease requiring surgery among hospitalised AS patients and significantly lower odds with IBD. Previous studies showed an association with age at onset, delay in diagnosis, bilateral involvement, axial/ enthesial disease and severe sacroiliitis. Some of these associations could not be analysed in our study due to lack of individual level patient data. Interestingly, epidemiological factors like smoking and obesity which have been linked to severe disease in PsA, were not found to have any significant association. Routine clinical hip exam and radiological imaging might help to identify high-risk patients. Early therapeutic strategies might be indicated for this specific population to prevent severe hip disease and need for hip replacement surgery.

Disclosure of Interest: None declared

DOI: 10.1136/annrheumdis-2018-eular.5031

\section{FRI0193 PROGNOSTIC MARKERS IN AXIAL SPONDYLOARTHRITIS (PROMISE) - CROSS SECTIONAL EVALUATION OF SERUM BIOMARKERS IN AXSPA, MECHANICAL BACK PAIN AND HEALTHY CONTROLS}

E. Reilly ${ }^{1}$, C. Fisher ${ }^{2}$, R. Sengupta ${ }^{1} .{ }^{1}$ Royal National Hospital for Rheumatic Diseases, Bath; ${ }^{2}$ University College London, London, UK

Background: In recent years there has been increasing interest in biomarkers in axial spondyloarthritis, for diagnosis, disease prognostication, and to monitor treatment effect. $^{12}$ Many biomarkers have been evaluated, but the role each of these plays and how they may interact is unclear.

Objectives: Our aim was to evaluate a broad panel of serum biomarkers in a large mixed cohort of patients, with Ankylosing Spondylitis (AS), non radiographic axial Spondyloarthritis ( $\mathrm{nr}-\mathrm{axSpA}$ ), mechanical back pain (MBP) and healthy controls $(\mathrm{HC})$, in order to identify any potential biomarkers for diagnosis by assessing the differences between the groups.

Methods: Cross sectional evaluation of 46 serum biomarkers was undertaken by Myriad RBM using multiplexed immunoassays of Multi-Analyte Panels, in a cohort of patients from a tertiary referral centre, consented as part of the Bath Spondyloarthritis BioBank. Validated patient reported outcomes (including BASDAI, $\mathrm{BASFI}$ ) and BASMI were completed. $50 \mathrm{HC}$ blood samples were also collected at University College London for biomarker analysis.

Results: 331 patients were included in the study, of which $59.5 \%$ AS, $8.2 \% \mathrm{nr}-$ axSpA, $15.7 \%$ mechanical back pain, $15.1 \%$ HC. $64.7 \%$ were male, mean age 44.2 years (SD 16.6), mean disease duration in the AS group of 22.4 years (SD 13.6) with $84 \%$ HLA B27 positive.
IL1 alpha and beta, IL1 receptor antagonist, IL2, 3, 4, 5, 7, 10, 15, 17, IL12 subunit p70, factor VII, GMCSF, IFN gamma, MMP9, TNF beta were the only biomarkers not to show statistical differences across the diagnostic groups (table 1.). 12 biomarkers showed a statistical difference between genders (table 1, column 1, p value significance indicated with ${ }^{*}<0.05,{ }^{* *}<0.01$ using Mann Whitney $U$, in addition to Factor $\mathrm{VII}{ }^{*}$ ).

Abstract FRI0193 - Table 1. Statistically significant serum biomarker results by diagnosis

\begin{tabular}{|c|c|c|c|c|c|}
\hline $\operatorname{Mean}(\mathrm{SO})$ & AS & $n r-2 k S_{Q A}$ & $\begin{array}{l}\text { Mechanical } \\
\text { backpain }\end{array}$ & $\begin{array}{l}\text { He althy } \\
\text { controls }\end{array}$ & $\begin{array}{l}\text { P value using } \\
\text { Kruskal Wallis }\end{array}$ \\
\hline $\mathrm{N}$ & 197 & 27 & 52 & 50 & \\
\hline Alpha 1 agti tryosio mg/ml & $3.2(1.2)$ & $2.3(0.8)$ & $2.1(0.8)$ & $1.4(0.4)$ & $<0.001$ \\
\hline Alpha 2 macroglobulin, $\mathrm{mg} / \mathrm{ml}$ & $4.0(1.4)$ & $3.7(1.2)$ & $3.6(1.5)$ & $3.1(1.9)$ & $<0.001$ \\
\hline *eta 2 macroglobulin, yl/ml & $3.4(2.7)$ & $2.2(0.8)$ & $2.0[0.9)$ & $1.6(0.3)$ & $<0.001$ \\
\hline $\begin{array}{l}\text { Brain derived Neucotcoplaic Factor, } \\
\mathrm{ng} / \mathrm{ml}\end{array}$ & $28.4(8.3)$ & $\frac{1.2(0.07}{30.3(8.2)}$ & $\frac{2.0(3.9)}{28.9(7.2)}$ & $21.0(4.5)$ & $<0.001$ \\
\hline CReactive Protein, y $\mathrm{g} / \mathrm{ml}$ & $29.6(69.1)$ & $4.6(9.2)$ & $4.7(9.0)$ & $3.0(4.4)$ & $<0.001$ \\
\hline Complement $\mathrm{C} 3, \mathrm{mg} / \mathrm{ml}$ & $2.8(1.3)$ & $1.9(0.9)$ & $1.8(1.0)$ & $0.8(0.2)$ & $<0.001$ \\
\hline " Eetaxia $1, \mathrm{DE} / \mathrm{ml}$ & $374.1(193.5)$ & $\begin{array}{l}241.4 \\
(174.8) \\
\end{array}$ & $\begin{array}{l}241.4 \\
(161.2)\end{array}$ & $170.1(1235)$ & $<0.001$ \\
\hline "Ferritin, ng/ml & $248.5(231.2)$ & $\begin{array}{l}177.9 \\
(177.3)\end{array}$ & $\begin{array}{l}\frac{(01.2)}{205.4} \\
(212.0)\end{array}$ & $89.9(74.9)$ & $<0.001$ \\
\hline "Fibrinogen, mg/ml & $0.0(0.0)$ & $0.0(0.0)$ & 0.0 & $0.0(0.0)$ & $<0.001$ \\
\hline Heotoglobin, mg/ml & $4.0(4.2)$ & $2.5(1.4)$ & $1.6(1.3)$ & $1.6(0.8)$ & $<0.001$ \\
\hline $\begin{array}{l}\text { Inte rcellular Adhesion Molecule 1, } \\
\mathrm{ng} / \mathrm{ml}\end{array}$ & $175.2(81.5)$ & $149.6(61.4)$ & $154.5(84.2)$ & $98.8(22.3)$ & $<0.001$ \\
\hline $\mathrm{ILG}, \mathrm{gg} / \mathrm{ml}$ & $2.4(6.9)$ & $0.3(1.4)$ & $0.7(1.6)$ & & $<0.001$ \\
\hline $118,0 \mathbb{R} / \mathrm{ml}$ & $33.4(69.6)$ & $\begin{array}{l}329.7 \\
(1484.9)\end{array}$ & $52.7(127.0)$ & $4.4(7.1)$ & $<0.001$ \\
\hline IL12 subunit $40, \mathrm{ng} / \mathrm{ml}$ & $0.4(0.2)$ & $0.4(0.2)$ & $0.3(0.3)$ & $0.3(0.2)$ & 0.001 \\
\hline 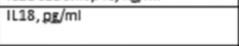 & $321.8(157.6)$ & $\begin{array}{l}295.1 \\
(171.5)\end{array}$ & $\begin{array}{l}281.8 \\
(204.3)\end{array}$ & $146.2(60.8)$ & 80.001 \\
\hline$\cdot|L 23, n g / m|$ & $0.8(1.0)$ & $0.3(0.7)$ & $0.3(0.7)$ & $0.1(0.4)$ & 80.001 \\
\hline $\begin{array}{l}\text { Macrophage Inflammatory Protain } \\
1 \mathrm{alpha}, \mathrm{gg} / \mathrm{ml}\end{array}$ & $23.1(62.1)$ & $20.2(41.2)$ & $24.2(37.6)$ & 0.0 & 0.005 \\
\hline $\begin{array}{l}\text { Macrophage Inflammatory Protein } \\
1 \text { beta, } \mathrm{pg} / \mathrm{ml}\end{array}$ & $678.5(595.4)$ & $\begin{array}{l}604.0 \\
(382.2)\end{array}$ & $\begin{array}{l}618.3 \\
(447.8)\end{array}$ & $290.8(192.3)$ & $<0.001$ \\
\hline $\begin{array}{l}\text { Matrix Metalloproteinase 3, } \\
\mathrm{ng} / \mathrm{ml}\end{array}$ & $24.2(15.9)$ & $11.6(4.6)$ & $14.1(8.3)$ & $13.5(6.7)$ & $<0.001$ \\
\hline 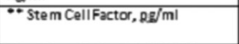 & $550.1(222.6)$ & $\begin{array}{l}362.6 \\
(259.0)\end{array}$ & $\begin{array}{l}389.1 \\
(235.5)\end{array}$ & $173.2(164.9)$ & $<0.001$ \\
\hline $\begin{array}{l}\text { Teell Specific Prote in RANTES, } \\
\mathrm{ng} / \mathrm{ml}\end{array}$ & $26.5(15.1)$ & $\frac{25.0(16.8)}{25.0(1)}$ & $21.2(10.5)$ & $20.4(7.6)$ & 0.03 \\
\hline $\begin{array}{l}\text { "Tissue Inhibitor of } \\
\text { Metallepcoteinases } 1, \mathrm{ng} / \mathrm{ml}\end{array}$ & $262.1(94.4)$ & $197.9(55.8)$ & $186.7(44.9)$ & $141.7(32.0)$ & $<0.001$ \\
\hline TNF alpha, pr/ml & $13.3(30.2)$ & $8.9(30.3)$ & $0.6(4.3)$ & 0.0 & $<0.001$ \\
\hline TNF receptor 2,ng/ml & $298.2(740.4)$ & $92.9(433.1)$ & $9.7(5.2)$ & $5.8(1.8)$ & 80.001 \\
\hline $\begin{array}{l}\text { - Vascular Cell Adhesion Molecule } \\
1, \mathrm{ng} / \mathrm{ml}\end{array}$ & $\begin{array}{l}1093.0 \\
(465.3)\end{array}$ & $\begin{array}{l}931.6 \\
(307.9)\end{array}$ & $\begin{array}{l}965.9 \\
(391.9)\end{array}$ & $651.0(141.1)$ & $<0.001$ \\
\hline $\begin{array}{l}\text { Vascular Endothelial Growh } \\
\text { Factor, ag/ml }\end{array}$ & $493.8(359.1)$ & $\begin{array}{l}439.6 \\
(316.8)\end{array}$ & $\begin{array}{l}394.1 \\
(215.4)\end{array}$ & $261.7(168.6)$ & $<0.001$ \\
\hline Vitamin D Binding Protein, $y \mathrm{~g} / \mathrm{ml}$ & $538.1(233.7)$ & \begin{tabular}{|l|}
414.6 \\
$(186.6)$ \\
\end{tabular} & $\begin{array}{l}439.3 \\
(227.9)\end{array}$ & $334.4(135.1)$ & $<0.001$ \\
\hline Von Willebrand Factor, yg/n & $161.2(89.9)$ & $110.6(51.9)$ & $109.2(77.6)$ & $95.5(51.1)$ & 80.001 \\
\hline $\begin{array}{l}\text { Monocyte Chemotactic Protein 1, } \\
\mathrm{gg} / \mathrm{ml}\end{array}$ & $484.7(220.0)$ & $\begin{array}{l}524.9 \\
(369.2)\end{array}$ & $\begin{array}{l}396.5 \\
(174.9)\end{array}$ & $314.7(133.6)$ & $<0.001$ \\
\hline
\end{tabular}

Conclusions: Serum biomarkers have been shown to vary with gender and diagnosis. Further work is planned to evaluate their relationship to disease activity using outcome measures such as the BASDAI, and radiographic scoring, to bette understand the role of each factor and combination of factors, and any causal link.

\section{REFERENCES}

[1] Maksymowych WP. An update on biomarker discovery and use in axia spondyloarthritis. Expert Rev Mol Diagn [Internet]. 2017 Nov;217(11):96574. [cited 2017 Dec 7]. Available from: https://www.tandfonline.com/doi/full/ 10.1080/14737159.2017.1381562

[2] Reveille JD. Biomarkers for diagnosis, monitoring of progression, and treatment responses in ankylosing spondylitis and axial spondyloarthritis. Clin Rheumatol [Internet] 2015 Jun 5;34(6):1009-18. [cited 2017 Jul 10]. Available from: http://link.springer.com/10.1007/s10067-015-2949-3

Acknowledgements: This study was undertaken as part of an ongoing piece of work that is being funded by Celgene.

Disclosure of Interest: None declared

DOI: 10.1136/annrheumdis-2018-eular.6740

\section{FRI0194 ANKYLOSING SPONDYLITIS DISEASE ACTIVITY SCORE (ASDAS) BASED ON A QUICK QUANTITATIVE CRP ASSAY PERFORMS SIMILARLY WELL TO ASDAS BASED ON CONVENTIONAL CRP IN PATIENTS WITH AXIAL SPONDYLOARTHRITIS}

F. Proft ${ }^{1}$, B. Muche ${ }^{1}$, M. Schmidt ${ }^{1}$, L. Spiller ${ }^{1}$, V. Rios Rodriguez ${ }^{1}$, J. Rademacher ${ }^{1}$, A.-K. Weber ${ }^{1}$, S. Lüders ${ }^{1}$, M. Protopopov ${ }^{1}$, I. Spiller ${ }^{1}$, J. Sieper ${ }^{1}$, D. Poddubnyy ${ }^{1,2}$. ${ }^{1}$ Charité - Universitätsmedizin Berlin; ${ }^{2}$ German Rheumatism Research Centre, Berlin, Germany

Background: The Ankylosing Spondylitis Disease Activity Score (ASDAS) is a composite index to assess disease activity in patients with axial spondyloarthritis (axSpA). According to the treat-to-target (T2T) recommendations for SpA, and the 